\title{
The role of competition versus cooperation in microbial community coalescence
}

\author{
Pablo Lechón ${ }^{1,2^{*}}$, Tom Clegg ${ }^{2}$, Jacob Cook ${ }^{2,3}$, Thomas P. Smith², Samraat Pawar ${ }^{2}$ \\ 1 Department of Ecology \& Evolution, University of Chicago, Chicago, IL, USA. \\ 2 Department of Life Sciences, Imperial College London, Silwood Park, Ascot, UK \\ 3 Centre for Integrative Systems Biology and Bioinformatics, Imperial College London, \\ UK \\ *plechon@uchicago.edu
}

\begin{abstract}
New microbial communities often arise through the mixing of two or more separately assembled parent communities, a phenomenon that has been termed "community coalescence". Understanding which features of complex parent communities determine the outcomes of any given coalescence event is an important challenge. While recent work has begun to elucidate the role of competition in coalescence, the effect of specific underlying structures of interactions remains unclear, and that of cooperation, a key interaction type commonly seen in microbial communities, is still largely unknown. Here, using a general consumer-resource model we study the combined effects of competitive and cooperative interactions on the outcomes of coalescence events. We simulate coalescence between pairs of communities lying on the spectrum from competition for shared carbon resources, to cooperation through cross-feeding on leaked metabolic by-products (facilitation). Specifically, we develop novel metrics to quantify community-wide competition and cooperation levels, and show that these can predict which community dominates in pairwise coalescence events. We find that when both types of interactions are present in the parent communities, the less competitive one, which maximizes resource partitioning, contributes a higher proportion of species to the new community after coalescence, regardless of its cooperativeness. However, counter-intuitively, when competition in both parent communities is significantly weaker than facilitation, the more cooperative one is at a disadvantage during coalescence because multi-species invasions are able to disrupt established cross-feeding links. Encounters between microbial communities are becoming increasingly frequent across the globe, and there is great interest in how the coalescence of microbial communities affects environmental and human health. Our study provides new insights into the mechanisms behind microbial community coalescence, and a framework to predict outcomes based on the interaction structures of parent communities.
\end{abstract}

\section{Author summary}

In nature, new microbial communities often arise from the fusion of whole, previously separated communities (community coalescence). Despite the crucial role that the interactions among microbial communities can play in ecosystems, our ability to predict the outcomes of coalescence events remains limited. Here, using a general mathematical model, we study whether and how the structure of species interactions confers an 
advantage upon a microbial community when it encounters another. We find that when both competition and cooperation are present, less competitive communities, which partition resources well, dominate in coalescence events. However, when competition is negligible, cooperation turns out to be detrimental to coalescence success, because highly cooperative communities are more susceptible to multi-species invasions. There are many potential environmental and health implications of microbial community coalescence, which will benefit from the theoretical insights that we offer here about the fundamental mechanisms underlying this phenomenon.

\section{Introduction}

Microbial communities are widespread throughout our planet [1], from the the human gut to the deep ocean, and play a critical role in natural processes ranging from animal development and host health 2, 3 , to biogeochemical cycles [4]. These communities are very complex, typically harbouring hundreds of species [5], making them hard to characterize. Recently, DNA sequencing has allowed a high-resolution mapping of these communities, opening a niche for theoreticians and experimentalists to collaboratively decipher their complexity and assembly 6 [14].

Unlike in the macroscopic world, entire, distinct microbial communities are often displaced over space and come into contact with each other due to physical (e.g., dispersal by wind or water) and biological (e.g., animal-animal interactions or leaves falling to the ground) factors $15 \sqrt{18}$. The process by which two or more communities that were previously separated join and reassemble into a new community has been termed community coalescence [19]. Although microbial community coalescence is likely to be common, the effects of the interactions structure of parent communities on the outcome of such events remains poorly understood [20].

Early mathematical models of community-community invasion in animals and plants revealed that when two communities merge after barrier removal, asymmetrical dominance of one community over the other one is likely to occur 21.22. As an explanation for this observation, it was argued that, because communities have been assembled through a history of competitive exclusion, they are likely to compete with each other as coordinated entities, rather than as a random collection of species. This result has been established more rigorously in recent theoretical work, where consumer-resource models have been used to show that in microbial community coalescence events, the winning community will be that which is capable of simultaneously depleting all resources more efficiently 23]. Overall, these findings suggest that communities arising from competitive species sorting exhibit sufficient "cohesion" to prevent successful invasions by members of other communities.

However, empirical support for the role of competition alone in coalescence is circumstantial, and the role of cooperation, which is commonly observed in microbial communities, remains largely unknown. For example, during coalescence in methanogenic communities, cohesive units of taxa from the community with the most efficient resource are co-selected 24], and in aerobic bacterial communities, the invasion success of a given taxon is determined by its community members [25]. Nonetheless, neither of these studies was able to establish the role of competition vs. cooperation in shaping cohesiveness, and coalescence success. Yet, these microbial communities exhibit cooperation through a dense cross-feeding network, where leaked metabolic by-products of one species act as resources for others 2628 . Indeed, several studies have suggested that a combination of competitive and cooperative interactions may determine the outcome of coalescence in microbial communities 29 31.

Here, we focus on the gap in our understanding of the relative importance of competition and cooperation in community coalescence. We use a consumer resource 
model that includes cross-feeding to assemble complex microbial communities spanning a broad range in the competition-cooperation spectrum. Using novel metrics, we then quantify community-level competition and cooperation in the assembled communities, as well as their "cohesiveness". Using the latter metric, we then determine the relative importance of the two types of interactions on success in pairwise coalescence events.

\section{Methods}

\section{Mathematical model}

We use a mathematical model based on Marsland et al. 6] (see Supporting text section 11) for the microbial consumer-resource dynamics (Fig 1):

$$
\begin{aligned}
\frac{d n_{\alpha}}{d t} & =g_{\alpha} n_{\alpha}\left((1-l) \sum_{j} c_{\alpha j} R_{j}-z_{\alpha}\right), \\
\frac{d R_{j}}{d t} & =\kappa_{j}-\sum_{\alpha} n_{\alpha} c_{\alpha j} R_{j}+l \sum_{\alpha k} n_{\alpha} D_{k j} c_{\alpha k} R_{k} .
\end{aligned}
$$

Here, $n_{\alpha}(\alpha=1, \ldots, s)$ and $R_{j}(j=1, \ldots, m)$ are the biomass abundance of the $\alpha^{t h}$ microbial (e.g., bacterial) species and the concentration of the $j^{\text {th }}$ resource (e.g., carbon source). The growth of species $\alpha$ is determined by the resources it harvests minus the cost of maintenance (two terms in the brackets). Resource uptake depends on the resource concentration in the environment $R_{j}$, and whether or not the species $\alpha$ uses resource $j\left(c_{\alpha j}=1\right.$ or $c_{\alpha j}=0$, respectively). The leakage term $l$ determines the proportion of this uptake that is released back into the environment as metabolic by-products, with the remainder $(1-l)$ being allocated to growth. The maintenance cost term, $z_{\alpha}$, is dependent on the number of resources that the $\alpha^{\text {th }}$ species consumes, and is given by the expression:

$$
z_{\alpha}=\chi_{0}(1+\epsilon) \sum_{j} c_{\alpha j}
$$

where $\chi_{0}$ is the average cost of being able to consume a given resource, the summation represents the total number of resources that species $\alpha$ is able to process, and $\epsilon$ is a random fluctuation sampled from a truncated (so that $z_{\alpha}>0$ ) normal distribution. $\mathrm{Eq} 2$ ensures that neither generalists nor specialists are systematically favoured during the community assembly by imposing a greater cost on species that consume a wider range of resources (see Supporting text section 2). The uptake that remains after subtracting this maintenance is transformed into biomass with a proportionality constant of $g_{\alpha}$, the value of which does not affect the results presented here.

The change in the concentration of resources in the environmental pool are determined by three terms. The first term represents the external supply $\kappa_{j}$, which gives the rate at which the $j^{\text {th }}$ resource enters the system. The second term is the uptake of the $j^{t h}$ resource from the environment, summed across all $s$ consumers in the system and the third term represents resources entering the environmental pool via leakage of metabolic by-products. By-product leakage is determined by the metabolic matrix $D$ (or the "stoichiometric" matrix; [6]), with the $j k^{\text {th }}$ element representing the leaked proportion of resource $j$ that is transformed into resource $k$. Due to conservation of energy, $D$ is, by definition, a row stochastic matrix, meaning that its rows sum to 1 .

The above model entails the following assumptions: (i) all resources contain the same amount of energy (taken to be 1 for simplicity), (ii) a type I functional response, (iii) binary consumer preferences, (iv) a shared core metabolism encoded in $D$, (v) a 
common leakage fractions for all species and resources, and (vi) a complex environment where all resources are externally supplied in equal amounts. We address the implications of these assumptions in the Discussion section.

\section{Competition and facilitation metrics}

Previous work suggests that during coalescence events, sets of species from the same community act as cohesive units and are selected together (ecological co-selection) 21 25. Our goal is to quantify community-level cohesion $(\Theta)$, by considering the interactions between all species in the community, classifying cooperative interactions as cohesion inducing, and competitive ones as cohesion precluding [35], so that

$$
\Theta=\mathcal{F}-\mathcal{C}
$$

where $\mathcal{F}$ and $\mathcal{C}$ are measures of community-wide facilitation and competition respectively, which we now define.

In the system described by Eqs 1 facilitation occurs when a species leaks metabolic by-products that are used by another species. We compute community-level cooperation by calculating the strengths of facilitation between individual species pairs and then averaging them across the community:

$$
\mathcal{F}=\left\langle F_{\alpha \beta}\right\rangle_{\alpha \neq \beta} .
$$

Here, $\mathcal{F}$ is a unitless quantity where the facilitation between the species, $\alpha \rightarrow \beta$ is given by:

$$
F_{\alpha \beta}=l \sum_{j k} \tilde{\kappa}_{j} c_{\alpha j} D_{j k} c_{\beta k},
$$

where $l$ is the strength of cooperative links, and the term $c_{\alpha j} D_{j k} c_{\beta k}$ represents the necessary condition to establish a cooperative link (see Supporting text section 3 for further details).

Competition for resources exists because of the overlap in resource preferences (the $c_{\alpha j}$ 's) between species. The realized strength of competition between species depends on the resource environment they experience, which is made up of two sources; the externally supplied resources, and the metabolic by-products generated by the community. Similar to facilitation, we develop a new metric of pairwise competition strength that accounts for both these sources. We then measure community-level competition by taking the average of the strengths of competition between all species pairs (see Supporting text section 3 for details), that is

$$
\mathcal{C}=\left\langle\left(C_{a}\right)_{\alpha \beta}+\left(C_{b}\right)_{\alpha \beta}\right\rangle_{\alpha \neq \beta} .
$$

Here again, community-level competition $\mathcal{C}$ is a unitless quantity partitioned into two components: $\left(C_{a}\right)_{\alpha \beta}$ measures the level of competition between species pair $(\alpha, \beta)$ for externally supplied resources, and $\left(C_{b}\right)_{\alpha \beta}$ the level of competition for resources that have been leaked by species across the community.

We define the competition for externally supplied (abiotically-generated) resources $\left(C_{a}\right)_{\alpha \beta}$ to be

$$
\left(C_{a}\right)_{\alpha \beta}=(1-l) \sum_{k} \tilde{\kappa}_{k} c_{\alpha k} c_{\beta k} .
$$

That is, intrinsic competition between the species pair is quantified by their common resource preferences through the scalar product of their preference vectors. Interaction strength is determined by the fraction of externally supplied resources that is effectively consumed, $1-l$, and the factor $\tilde{\kappa}_{k}$ accounts for possible differences in external supply rate between resources. 


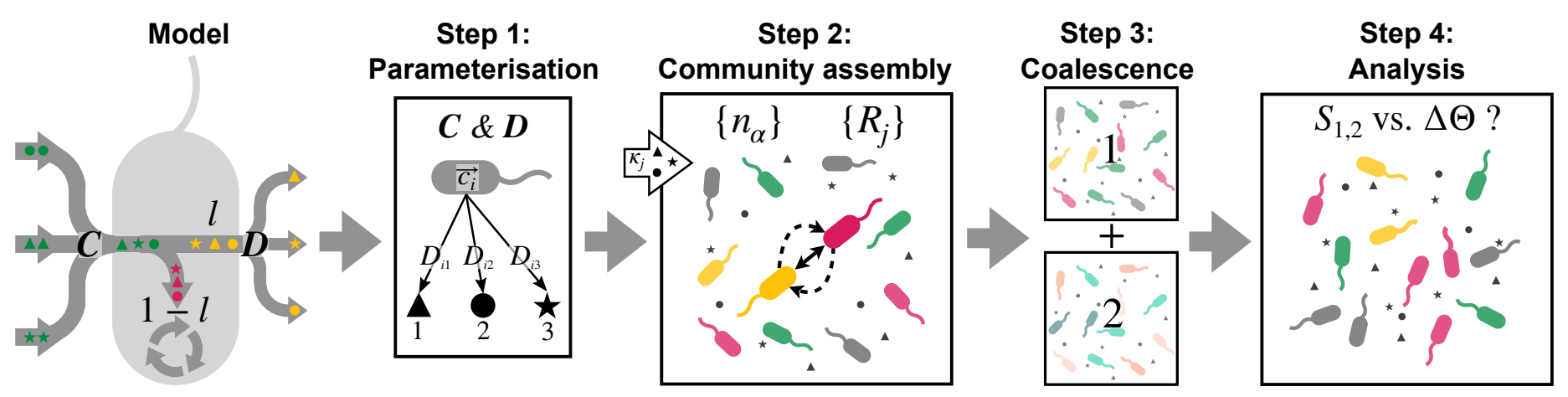

Fig 1. Overview of the coalescence modelling methodology. Step 1. The matrix of resource preferences $(C)$ and the metabolic matrix $(D)$ are sampled for each community under both the unstructured, and structured scenarios. Black polygons are different resource types. Step 2. Dynamics of the system are allowed to play out (Eqs 1) until steady state is reached. The solid bidirectional arrow represents a competitive link between two species, and the unidirecitonal dashed arrows capture facilitation from one species to another. Step 3. Pairs of the assembled parent communities are randomly picked, mixed, and the resulting community re-run to steady state. Step 4 . The contribution of each parent community to the final mix is analyzed $\left(S_{1,2}, \mathrm{Eq} 9\right)$ as a function of their cohesion before they coalesced $(\Delta \Theta, \mathrm{Eq} 3)$.

The second term in Eq 6 corresponds to competition for resources leaked as metabolic by-products (biotically-generated resources), and is written as

$$
\left(C_{b}\right)_{\alpha \beta}=l \sum_{j k} \tilde{\kappa}_{j} D_{j k}\left(c_{\alpha j}+c_{\beta j}\right) c_{\alpha k} c_{\beta k} .
$$

Here, $l$ is the strength of competition on leaked resources (the rationale behind this can be found in Supporting text section 3 and Fig S1), and the product

$D_{j k}\left(c_{\alpha j}+c_{\beta j}\right) c_{\alpha k} c_{\beta k}$ represents the necessary conditions to have effective competition for the $k^{\text {th }}$ leaked resource (see Supporting text section 3 for details).

\section{Simulations}

Fig 1 presents an overview of our methodology used to simulate community coalescence, which we describe here. The matrix implementation used in the actual simulations is described in the Supporting text section 8 .

\section{Step 1: Parameterization}

We first generate parent communities with interactions across the spectrum of competition to cooperation. To this end, for each parent community, resource preferences and secretion parameters (the $c_{\alpha j}$ 's and $D_{j k}$ 's, respectively) of $s=m=60$ consumer species and resource types are sampled from random distributions, but with two types of constraints that modulate the competition and facilitation levels (cohesiveness) achieved at steady state, or introduce structure in the system.

Modulating competition and facilitation levels. We use an iterative procedure to impose a specific level of competition by increasing or decreasing niche similarity between consumers (see Supporting text section 4). In this procedure, resource preferences of single species are assigned iteratively by re-evaluating the probability that species $\alpha$ samples resource $j$, given by

$$
p_{\alpha j}=\left(1-k_{c}\right) \frac{1}{m}+k_{c} \tilde{d}_{\alpha-1 j},
$$




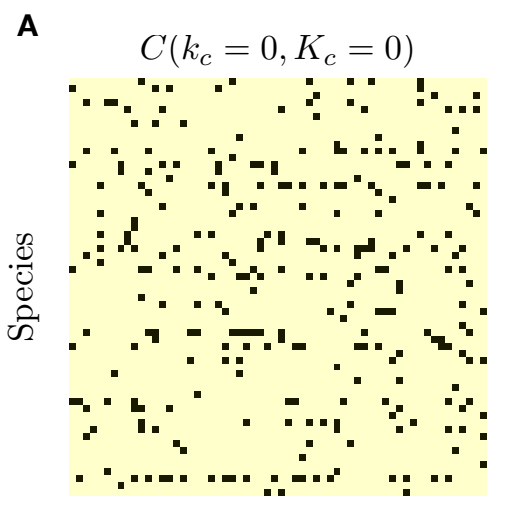

B

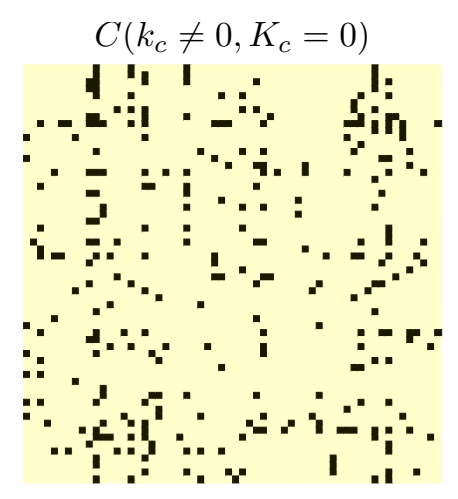

Resource type

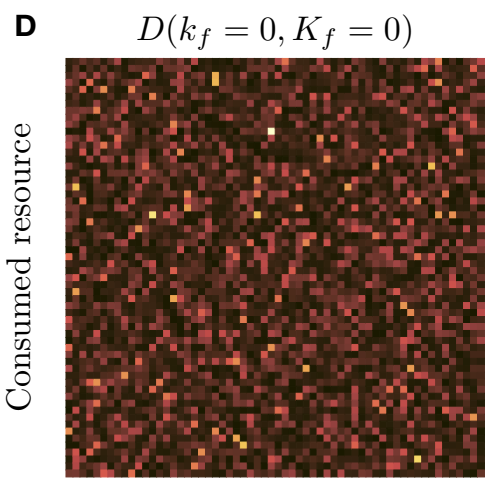

E

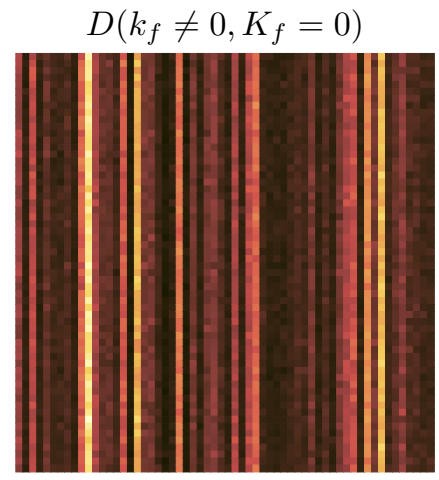

C

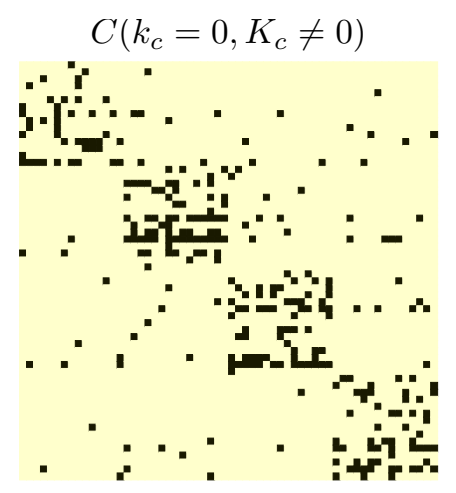

$\mathbf{F}$

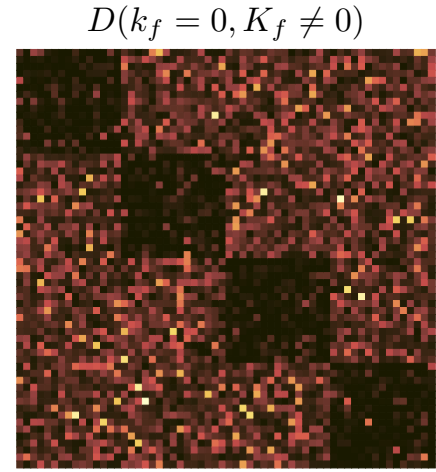

Metabolic by-product

Fig 2. Examples of differently structured preference $(C)$ and metabolic $(D)$ matrices. These have been generated with different combinations of the competition and facilitation parameters $k_{c}, K_{c}, k_{f}$, and $K_{f}$ in systems of 60 resource types and 60 consumer species. In the metabolic matrices (D-F), lighter colours indicate higher values (resource fractions secreted). A \& D: Purely random matrices, where all the four parameters are 0. B \& E: As $k_{c}$ and $k_{f}$ are increased the regime moves towards greater preferential feeding, where more demanded resources are more likely to be consumed (increase of $k_{c}$ ), but also secreted at higher fractions (increase of $k_{f}$ ). $\mathbf{C}$ \& F: Instead, if $K_{c}$ and $K_{f}$ are increased, the regime moves towards more structured resource use, where species in one guild are more likely to consume resources from their preferred resource class (increase in $K_{c}$ ), and leak higher fractions of resources that do not belong to their preferred resource class (increase in $K_{f}$ ).

where $m$ is the number of resource types, $\tilde{d}_{\alpha-1 j}$ is the normalized cumulative demand of resource $j$ at iteration $\alpha-1$, and $k_{c}$ is the competitiveness factor (see Supporting text section 4 for details). In each step of the iteration, the sampling probability of each resource by a given consumer is changed according to the demand on it such that highly-demanded resources are more likely to be sampled in the next step ("preferential feeding"). Note that $k_{c}$ modulates how much consumers prefer highly-demanded resources, such that when $k_{c}=0$, the sampling is uniformly random (Fig 22A); as $k_{c} \rightarrow 1$, the feeding becomes increasingly preferential (Fig $2 \mathrm{~B}$ ).

The facilitation level of the community depends on the topology of metabolic matrix $D$ (Fig 2 $\mathrm{P}$ ), which is specified such that the resources that are highly demanded are also secreted in large fractions (Fig $2 \mathrm{E}$, and see Supporting text section 4 for details).

Introducing guild structure. Recent empirical studies suggests that microbial species tend to form guilds with similar metabolic capabilities, thus introducing some degree of functional redundancy in the communities they form 32,33 . Theoretical studies support these observations $7,14,34$. We therefore add further structure to the matrices 
$C$ and $D$, by partitioning resources into classes, and constraining consumers to feed on a preferred class, but leak to any other, forming consumer guilds. Adding this structure yields two interaction layers (imagine superimposing Figs $2 \mathrm{~B} \& \mathrm{C}$ with Figs $2 \mathrm{E} \& \mathrm{~F}$ ): inter-guild facilitation and competition between consumers preferring distinct resource classes, and intra-guild facilitation and competition, which stems from the previously-imposed preferential feeding.

Resource preferences in this scenario are assigned similarly to the unstructured preferential feeding above, except that the probability that species $\alpha_{A}$ (which feeds preferentially on resource class $A$ ) samples resource $j$, is now weighted up or down depending on whether $j$ belongs in guild $A$, or not, respectively (Fig $2 \mathrm{C}$, derivation in Supporting text section 5, as:

$$
p_{\alpha j}^{A}= \begin{cases}\frac{1}{\mathcal{A}}\left(1+K_{c}\left(N_{c}-1\right)\right)\left(\frac{1}{m}\left(1-k_{c}\right)+\tilde{d}_{\alpha-1 j} k_{c}\right) & \text { if } j \in A \\ \frac{1}{m}\left(1-K_{c}\right) & \text { otherwise. }\end{cases}
$$

Here $\mathcal{A}$ is a normalization constant ensuring that the probabilities sum to 1 . The magnitude of this effect is given by the constant $K_{c}$, which controls the amount of consumer guild structure in $C$.

The metabolic matrix $D(\mathrm{Fig} 2 \mathrm{2} \mathrm{F})$ is constructed such that the fraction of leaked by-product $k$ is lower if it belongs to the same class as the consumed resource $j$ (elements within block-diagonals of $D$ ), and higher otherwise (off-block diagonal elements of $D$ ). The prominence of this structure in the matrix is given by the inter-guild facilitation factor $K_{f}$ (see Supporting text section 5 for details).

\section{Step 2: Assembly of parent communities}

After parameterization, we numerically simulate the dynamics of each parent community (with $s=m=60$ ), according to $\mathrm{Eq} 1$ until steady state is reached. We assemble parent communities using the preferential feeding parameterization of the consumer preference and metabolic matrices either with or without the guild-structure described above (henceforth referred to as the structured or unstructured scenarios, respectively). Within each of the two sets, we perform 100 random assemblies at each combination of competition and facilitation values (i.e. $k_{c}=k_{f} \in[0,0.5,0.9]$ ), setting $K_{c}=K_{f}=0$ or $K_{c}=K_{f}=0.9$ for the unstructured and structured cases respectively. Traversing the parameter space in this way ensures that all regimes shown in Fig 2 and their possible superpositions are visited 100 times. In order to assess the effect of leakage (which will modulate interactions strength between species as shown by Eqs 6 \& 4 we repeat these simulations in three leakage regimes setting $l=[0.1,0.5,0.9]$. Further details of the assembly simulations are in Supporting text section 6 .

\section{Step 3: Coalescence}

To simulate coalescence we randomly sample pairs of assembled communities $\left(2 \cdot 10^{4}\right.$ pairs for each leakage level), set all resources to their initial concentrations, and numerically integrate the new combined system to steady state. By selecting random communities we sample across a range of interaction types within communities (i.e. from competition to cooperation) and differences in cohesiveness values between the two communities (see Supporting text section 6 for further details).

\section{Step 4: Post-coalescence analysis}

We analyse the contribution of the communities in the original pair to the species' presence or absence profile in the coalesced community, and how this contribution 
depends on the cohesiveness of the parent communities $\left(\Theta_{1}\right.$ and $\Theta_{2}$, measured before coalescence using Eq 3). We measure the similarity of the coalesced community to each of the two parents (indexed by 1 and 2), which gives a measure of parent community dominance:

$$
S_{1,2}=\overrightarrow{p_{f}} \cdot\left(\frac{\overrightarrow{p_{1}}}{r_{1}}-\frac{\overrightarrow{p_{2}}}{r_{2}}\right),
$$

where $\overrightarrow{p_{f}}, \overrightarrow{p_{1}}$, and $\overrightarrow{p_{2}}$ are $\left(s_{1}+s_{2}\right)$-dimensional vectors of species presence-absence in the post-coalescent, and parent communities 1 , and 2 , respectively, with $r_{1}$ and $r_{2}$ the species richness values of the parent communities 1 and 2, respectively (calculated as $r_{i}=\sum \overrightarrow{p_{i}}$ ). If $S_{1,2}=1$, the coalesced community is identical to parent community 1 , and if $S_{1,2}=-1$, it is identical to parent community 2 . This measure is independent of the number of consumer species, allowing us to mix communities with different levels of species richness, avoiding bias in similarity towards the richer one.

\section{Results}

\section{Minimizing competition ensures coalescence success}

We first simulate coalescence between pairs of communities in the unstructured scenario (i.e. without guild-structure; Fig $3 \mathrm{~A}$ ). We find that communities with higher cohesion values tend to perform better in coalescence as seen by the positive relationship between parent community dominance $\left(S_{1,2}\right)$ and the difference in cohesion between the parent communities $\left(\Theta_{1}-\Theta_{2} ; \operatorname{Fig} 3 \mathrm{C}\right)$. That is, communities that emerge following coalescence tend to have greater similarity with their more cohesive parent. This relationship holds across all three leakage regimes. At low leakage, facilitation is negligible (blue line in Fig $3 \mathrm{~B}$ ), and competition is mainly for abiotically-generated resources (dashed line in Fig $3 \mathrm{~B}$ ). Thus, in this regime, being more cohesive is equivalent to being less competitive and communities that minimize competition succeed in coalescence events. This trend holds at higher values of leakage, even where facilitation is larger than competition (now mainly for leaked resources) on average. This suggests that with only preferential feeding, (minimizing) competition drives the outcome of community coalescence, overriding the effects of facilitation (see Fig S5 for further details).

Previous work has linked success in coalescence to effective resource usage [23]. To test this in our system, we plot, for all the assembled communities, the total resource abundance per consumer at steady state, for three values of leakage, as a function of the community' intrinsic cohesion (Fig 3D), which is given by

$$
\hat{\Theta}=\frac{F-C_{b}}{l}-\frac{C_{a}}{1-l} .
$$

This measure accounts for just the cohesion structure, leaving out the strength of the links (allowing comparison between different values of leakage). The negative correlation we observe (which holds too for the guild-structured scenario, Fig S4) implies that as expected, more cohesive communities deplete resources more efficiently. As the value of leakage decreases, more diverse and less competitive communities (brighter colors and circular shapes, corresponding to decreasing $k_{c}$ values, in Fig 3D cluster together more compactly at lower depletion levels. This is because more competitive communities tend to be less diverse (see Fig S1), and below a certain richness threshold, are not able to saturate the available resource niches. Cooperative links in communities with low leakage are too weak to balance this. On the contrary, in high leakage communities, the stronger cooperative links are able to counter the effects of competition by making all resources more evenly available across the community. 
A

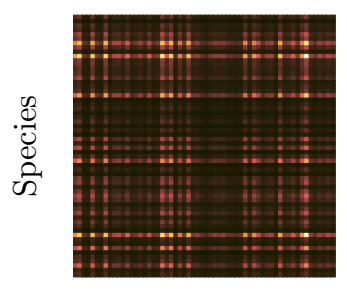

B

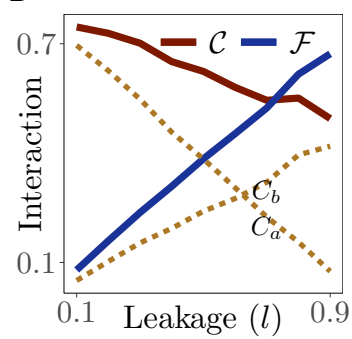

C

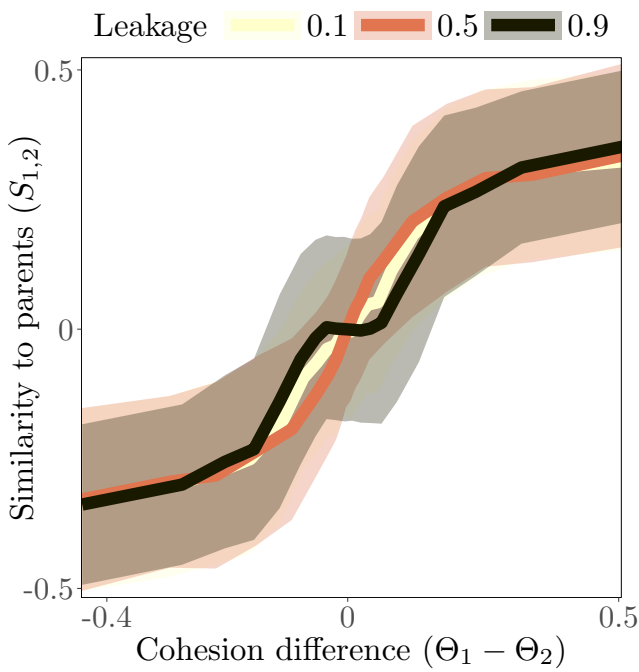

D

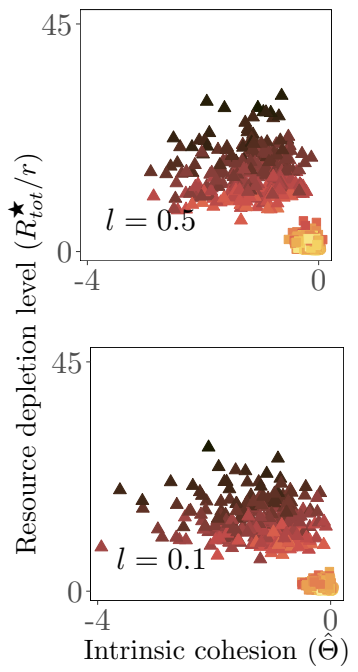

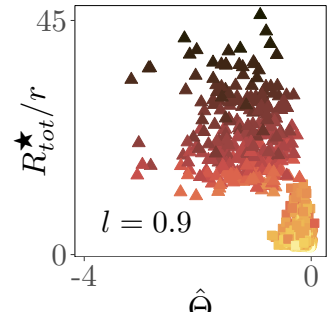

$\hat{\Theta}$

$k_{c} \quad \circ 0 \square 0.5 \triangle 0.9$

Species

richness 203040

Fig 3. Community coalescence with preferential feeding. A: Example of the secretion matrix of $(C D)_{\alpha k}$ 's, the total leakage of resource $k$ by species $\alpha$. B: Community-level competition $\mathcal{C}$ (dark red) and facilitation $\mathcal{F}$ (blue) averaged across simulations for each leakage value. Competition for abiotically-generated resources $\left(C_{a}\right)$ decreases, and that for biotically-generated resources $\left(C_{b}\right)$ increases with leakage, with total competition remaining consistently high throughout. Facilitation, on the other hand, increases linearly with leakage. C: The post-coalescence community is more similar to its more cohesive parent. Shown is the binned mean (20 bins) over communities with similar parent cohesion difference $\Theta_{1}-\Theta_{2}$ (solid line) \pm 1 standard deviation (shaded) for the three leakage values. D: Total resource abundance per consumer at steady state is negatively correlated with intrinsic cohesion, for all leakage values, confirming that more cohesive communities, which also tend to be more species-rich, and less competitive, deplete resources more efficiently. Different values of $k_{c}$ are in different shapes.

\section{Cooperation undermines coalescence success}

Next we simulate community coalescence by imposing consumer guild structure. This effectively results in the biotically-generated resource competition $\left(C_{b}\right.$ in Eq 6$)$ becoming very low, bringing out a new regime at high $l$ values where $\mathcal{C}<<\mathcal{F}$ (Fig $4 \mathrm{~B}$ ) When coalescence is simulated between such pairs of communities, we find that in the low leakage regime, where significant competition is present, the same result (yellow and red lines in Fig $4 \mathrm{C}$ ) as in the case with only preferential feeding (Fig $3 \mathrm{~B}$ ) is recovered. In the highest leakage regime the correlation between parent community dominance and cohesion reverses (black line, Fig $4 \mathrm{C}$ ). Competition is negligible in this regime (Fig $4 \mathrm{~B}$ ), so being more cohesive is equivalent to having higher levels of facilitation. Thus, the negative correlation indicates that more facilitative communities perform poorly in coalescence events, that is, cooperative communities are easily invaded by a randomly-picked community (see Fig S5). One empirically-supported explanation for this observation is that multi-species invasions can easily disrupt cooperative links 36 38. To test this, we measured the average facilitation in the mixed and losing communities, as well as in the group of species that went extinct during the coalescence event, across all simulations at each leakage value (Fig 4D). The results confirm our intuition: both, the species in the losing parent community, and the species that go extinct from that community, tend to be engaged in stronger cooperative links relative to those in the post-coalescence community (yellow and red bars are higher than the black bars). As expected, this pattern is strongest for very high values of leakage 
A

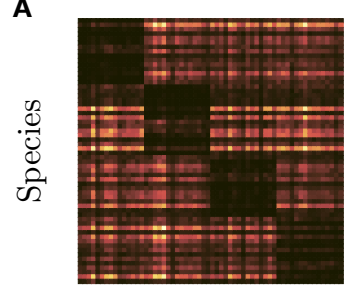

B

Metabolic by-product

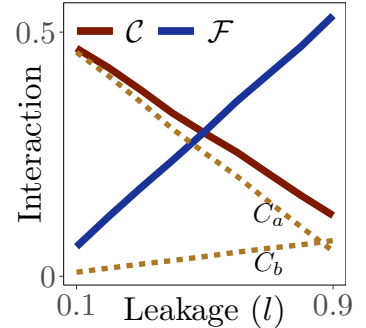

C

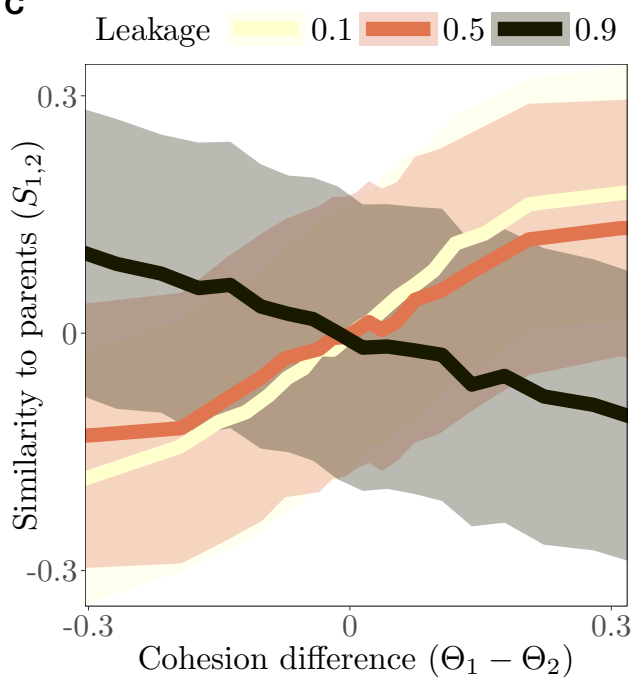

D

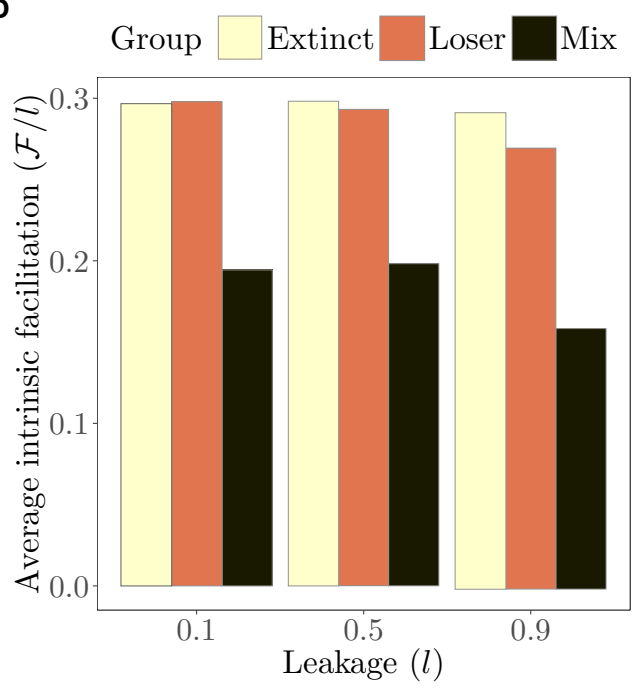

Fig 4. Community coalescence with consumer guilds present. A: Example of a secretion matrix with consumer guild structure in addition to preferential feeding. B: Community-level competition $(\mathcal{C})$ and facilitation $(\mathcal{F})$ averaged across simulations for different levels of leakage. For low values of leakage, competition for abiotically-generated resources $\left(C_{a}\right)$ dominates, and for high values of leakage facilitation $(\mathcal{F})$ is the important term. Competition for biotically-generated resources $\left(C_{b}\right)$ is consistently low due to the consumer guild structure imposed on matrices $C$ and $D$. C: Parent community dominance is positively correlated with $\Delta \Theta$ for low values of leakage, when $\mathcal{C}>\mathcal{F}$; but negatively correlated with $\Delta \Theta$ for high values of leakage, when $\mathcal{C}<\mathcal{F}$. $\mathbf{D}$ : The difference in average intrinsic facilitation between extinct species, losing parent community, and post-coalescence community, increases with leakage, indicating that in more cooperative communities, facilitation links are disproportionately disrupted by invading species.

$(l=0.9)$, where the strongest cooperative links are present. Note that the y-axis in Fig $4 \mathrm{D}$ is leakage-independent, and therefore it is only measuring the facilitation topology, that is, the number of cooperative links independent of the flux through them, $l$ (recall Eq 5 ).

\section{Discussion}

New microbial communities often emerge through community coalescence 19 . Previous studies have focused on the cohesiveness exhibited by coalescing communities $21,25,39$. In Tikhonov's study, the cohesion displayed by coalescing communities in the absence of cooperative interactions was explained in terms of effective resource depletion 23. This allowed the winning community to engineer an environment more favorable for itself than for the losing community, which was partially or completely displaced. This result has been experimentally verified in methanogenic communities 24], which are characterized by a dense metabolic cross-feeding network. However the question remains as to how a model built exclusively around competition can explain the complex reality of coalescence in the presence of extensive cross-feeding. Our findings offer new, general insights by explicitly considering the effect of the structure of interactions underlying resource competition and cooperation. In particular, we find that the balance between competition and cooperation can substantially change the outcome of coalescence events, as has been suggested recently 30 . Overall, these findings extend the results of previous theoretical studies to accommodate metabolic interdependence (cross-feeding); 
an essential feature of real microbial communities [35, 38], and also provide theoretical, mechanistic insights into empirical studies that have demonstrated the importance of cross-feeding interactions on coalescence 24 .

Reassuringly, the emerging pattern we find at $l \approx 0$ (yellow line in Fig $3 \mathrm{~B}$ ), parallels that emerging in 23]. However, we do not define cohesiveness in terms of resource depletion efficacy, but rather as a function of the underlying interaction structure, confirming that resource use efficiency is indeed a consequence of structural minimization of competition (Fig $3 \mathrm{D}$ ). The consistency of the trend in Fig $3 \mathrm{~B}$ across the whole leakage range suggests that minimizing competition is the main factor driving the outcome of community coalescence even in the presence of strong cooperation. When $l>0$, high competition levels prevailed, but were taking place in an altered environment, one engineered by a community that both consumed and secreted resources. Ultimately, competition for leaked resources exists because the species are secreting resources necessary for their own growth. While this might seem disadvantageous and thus unrealistic at first, leaking essential resources is an common phenomenon in microbial systems 40, 41], and may be advantageous as a "flux control" mechanism employed by individual cells to promote growth in crowded environments 42,43 .

After adding consumer guild structure to parent communities consistent with empirical observations, we saw a new regime emerge for high values of leakage where the community-level cooperation was high, and competition was negligible. In this case the consistent positive correlation between cohesion and coalescence success found previously was reversed (Fig 4D), driven by the fact that multi-species invasions can easily disrupt cross-feeding links in more cooperative communities. This result is in line with empirical results showing that cooperative links are susceptible to be intercepted by species invasions 36 38. Nonetheless, recent in-silico results of single species invasions on microbial communities have found that cooperative communities are more resistant to invasions than their competitive counterparts [44]. The apparent contradiction between this finding and our own suggests that invasions in the context of complex communities cannot be understood from the study of single species.

Investigating the dynamics of these multi-species invasions of cooperative communities in greater detail is a potentially important area for future work.

Throughout this work, we assumed that all resources were supplied, and at a fixed rate. Removing environmental fluctuations in the form of resource variation allowed us to focus on coalescence in terms of variation in species interaction structure alone.

While this assumption may be sensible in some cases [45, it is an oversimplification in others 31. Extending this theoretical framework to study the effects of resource supply variation, e.g., by allowing substrate diversification from a single supplied resource [6.7], is a promising direction for future research. In such cases, we expect that only the cooperative links necessary to diversify the supplied carbon source will persist upon coalescence events, but above that threshold, the results presented here would be recovered. The resource dynamics where further simplified in this work by assuming a type-I functional response. This is not expected to change our results substantially, since our simulations are performed at steady state, where all three types of functional responses behave the same. Given the heavy simulation based approach taken here, assuming a core metabolism and leakage common to the whole community, made the dynamics computationally tractable, while ensuring that the system was not far away from the conditions of real communities [6,34.

The pairs of coalescing communities in this work were drawn with no richness restrictions, that is, communities with different species richness were allowed to compete. Consequently, the results reported here are independent of the species richness of the mixed communities. Interestingly, several previous studies have pointed to microbial community diversity as an important factor driving resource use efficiency 
and, therefore, determining community resistance against biotic and abiotic perturbations [46 48]. These observations do not necessarily contradict the results reported here. Instead, our findings suggest that community interactions may be a more fundamental mechanism explaining the response of communities to environmental and biotic perturbations, and that biodiversity is rather a consequence of the underlying community interaction network. It is not surprising that empirical studies usually focus on biodiversity's influence on community stability (the "diversity-stability" relationship) rather than of community interactions, since the latter is much harder to measure than the former. Understanding biodiversity as an emergent property of the interaction network topology in a microbial community is a promising line of future research [49].

Encounters between microbial communities are becoming increasingly frequent [50], and mixing of whole microbial communities is gaining popularity for bio-engineering [51], soil restoration [52, faecal microbiota transplantation [53, 54], and the use of probiotics [55]. We present a framework which relates the nature of species interaction in microbial communities to the outcome of community coalescence events. Although more work is required to bridge the gap between theory and empirical observations, this study constitutes a key step in that direction.

\section{Supporting information}

Supporting text section 1 - Relationship with other consumer resource models.

Supporting text section 2 - Cost function.

Supporting text section 3 - Competition and Facilitation Metrics.

Supporting text section 4 - Modulating cohesion levels.

Supporting text section 5 - Adding consumer guild structure.

Supporting text section 6 - Further details on community assembly simulations.

Supporting text section 7 - Further details on community coalescence simulations.

Supporting text section 8 - Matrix representation of the model.

Fig S1 Mechanism of resource recycling

Fig S2 Consumer preferences before and after community coalescence

Fig S3 Heat-maps for each value of 1 of the average richness $\mathrm{r}$ as a function of $k_{c}$ and $k_{f}$.

Fig S4 Depletion level in the structured scenario.

Fig S5 Plots of each component of the cohesion measure for both the unstructured and structured scenarios. 
Fig S6 Plots of each component of the cohesion measure for all leakages in the unstructured scenario.

Fig S7 Plots of each component of the cohesion measure for all leakages in the unstructured scenario.

\section{Author Contributions}

Conceptualization: Pablo Lechón, Tom Clegg, Jacob Cook, Thomas P. Smith, Samraat Pawar

Formal Analysis: Pablo Lechón

Methodology Pablo Lechón

Software Pablo Lechón

Visualization: Pablo Lechón

Writing - Original Draft Preparation: Pablo Lechón

Writing - Review \& Editing: Pablo Lechón, Tom Clegg, Jacob Cook, Thomas P. Smith, Samraat Pawar.

Funding Acquisition: Samraat Pawar

\section{References}

1. Fierer N, Jackson RB. The diversity and biogeography of soil bacterial communities. Proceedings of the National Academy of Sciences of the United States of America. 2006;103(3):626-631. doi:10.1073/pnas.0507535103.

2. Huttenhower C, Gevers D, Knight R, Al E. Structure, function and diversity of the healthy human microbiome. Nature. 2012;486(7402):207-214. doi:10.1038/nature11234.

3. McFall-Ngai M, Hadfield MG, Bosch TCG, Carey HV, Domazet-Lošo T, Douglas AE, et al. Animals in a bacterial world, a new imperative for the life sciences. Proceedings of the National Academy of Sciences of the United States of America. 2013;110(9):3229-3236. doi:10.1073/pnas.1218525110.

4. Falkowski PG, Fenchel T, Delong EF. The microbial engines that drive earth's biogeochemical cycles. Science. 2008;320(5879):1034-1039.

doi:10.1126/science.1153213.

5. Gilbert JA, Jansson JK, Knight R. The Earth Microbiome project: Successes and aspirations. BMC Biology. 2014;12(69):12915-014. doi:10.1186/s12915-014-0069-1.

6. Marsland R, Cui W, Goldford J, Sanchez A, Korolev K, Mehta P. Available energy fluxes drive a transition in the diversity, stability, and functional structure of microbial communities. PLoS Computational Biology. 2019;15(2):e1006793. doi: 10.1371/journal.pcbi.1006793. doi:10.1371/journal.pcbi.1006793. 
7. Goldford JE, Lu N, Bajić D, Estrela S, Tikhonov M, Sanchez-Gorostiaga A, et al. Emergent simplicity in microbial community assembly. Science. 2018;361(6401):469-474. doi:10.1126/science.aat1168.

8. Goyal A, Maslov S. Diversity, Stability, and Reproducibility in Stochastically Assembled Microbial Ecosystems. Physical Review Letters. 2018;120(15):158102. doi: 10.1103/PhysRevLett.120.158102. doi:10.1103/PhysRevLett.120.158102.

9. Friedman J, Higgins LM, Gore J. Community structure follows simple assembly rules in microbial microcosms. Nature Ecology and Evolution. 2017;1(5):41559-017. doi:10.1038/s41559-017-0109.

10. Costello EK, Stagaman K, Dethlefsen L, Bohannan BJM, Relman DA. The application of ecological theory toward an understanding of the human microbiome. Science. 2012;336(6086):1255-1262. doi:10.1126/science.1224203.

11. Vila JCC, Jones ML, Patel M, Bell T, Rosindell J. Uncovering the rules of microbial community invasions. Nature Ecology and Evolution. 2019;3(8):1162-1171. doi:10.1038/s41559-019-0952-9.

12. Estrela S, Vila JCC, Lu N, Bajic D, Rebolleda-Gomez M, Chang CY, et al. Metabolic rules of microbial community assembly. bioRxiv. 2020. doi:10.1101/2020.03.09.984278.

13. Coyte KZ, Rao C, Rakoff-nahoum S, Foster KR. Ecological rules for the assembly of microbiome communities. PLOS Biol. 2021;19(2):e3001116. doi:10.1371/journal.pbio.3001116.

14. Lorenzo Fant, Iuri Macocco, Jacopo Grilli Eco-evolutionary dynamics lead to functionally robust and redundant communities bioRxiv. 2021; 1-12

15. Luo X, Xiang X, Yang Y, Huang G, Fu K, Che R, et al. Seasonal effects of river flow on microbial community coalescence and diversity in a riverine network. FEMS Microbiology Ecology. 2020;96(8). doi:10.1093/femsec/fiaa132.

16. Vass M, Székely AJ, Lindström ES, Osman OA, Langenheder S. Warming mediates the resistance of aquatic bacteria to invasion during community coalescence. Molecular Ecology. 2021;doi:10.1111/mec.15800.

17. Kort R, Caspers M, van de Graaf A, van Egmond W, Keijser B, Roeselers G. Shaping the oral microbiota through intimate kissing. Microbiome. 2014;2(1):1-8. doi:10.1186/2049-2618-2-41.

18. Evans SE, Bell-Dereske LP, Dougherty KM, Kittredge HA. Dispersal alters soil microbial community response to drought. Environmental Microbiology. 2020;22(3):905-916. doi:10.1111/1462-2920.14707.

19. Rillig MC, Antonovics J, Caruso T, Lehmann A, Powell JR, Veresoglou SD, et al. Interchange of entire communities: Microbial community coalescence. Trends in Ecology and Evolution. 2015;30(8):470-476. doi:10.1016/j.tree.2015.06.004.

20. Rillig MC, Lehmann A, Aguilar-Trigueros CA. Soil microbes and community coalescence. Pedobiologia. 2016;59(1-2):37-40. doi:10.1016/j.pedobi.2016.01.001.

21. Gilpin M. Community-level competition: Asymmetrical dominance. Proceedings of the National Academy of Sciences of the United States of America. 1994;91(8):3252-3254. doi:10.1073/pnas.91.8.3252. 
22. Toquenaga Y. Historicity of a simple competition model. Journal of Theoretical Biology. 1997;187(2):175-181. doi:10.1006/jtbi.1997.0428.

23. Tikhonov M. Community-level cohesion without cooperation. eLife. 2016;5:e15747. doi: 10.7554/eLife.15747. doi:10.7554/eLife.15747.

24. Sierocinski P, Milferstedt K, Bayer F, Großkopf T, Alston M, Bastkowski S, et al. A Single Community Dominates Structure and Function of a Mixture of Multiple Methanogenic Communities. Current Biology. 2017;27(21):3390-3395. doi:10.1016/j.cub.2017.09.056.

25. Lu N, Sanchez-gorostiaga A, Tikhonov M, Sanchez A. Cohesiveness in microbial community coalescence. bioRxiv. 2018; p. 282723 doi:10.1101/282723.

26. Hansen SK, Rainey PB, Haagensen JAJ, Molin S. Evolution of species interactions in a biofilm community. Nature. 2007;445(7127):533-536. doi:10.1038/nature05514.

27. Lawrence D, Fiegna F, Behrends V, Bundy JG, Phillimore AB, Bell T, et al. Species interactions alter evolutionary responses to a novel environment. PLoS Biology. 2012;10(5):e1001330. doi: 10.1371/journal.pbio.1001330. doi:10.1371/journal.pbio.1001330.

28. Embree M, Liu JK, Al-Bassam MM, Zengler K. Networks of energetic and metabolic interactions define dynamics in microbial communities. Proceedings of the National Academy of Sciences of the United States of America. 2015;112(50):15450-15455. doi:10.1073/pnas.1506034112.

29. Rivett DW, Jones ML, Ramoneda J, Mombrikotb SB, Ransome E, Bell T. Elevated success of multispecies bacterial invasions impacts community composition during ecological succession. Ecology Letters. 2018;21(4):516-524 doi:10.1111/ele.12916.

30. Castledine M, Sierocinski P, Padfield D, Buckling A. Community coalescence: An eco-evolutionary perspective. Philosophical transactions of the Royal Society of London Series B, Biological sciences. 2020;375(1798):20190252. doi:10.1098/rstb.2019.0252.

31. Albright MBN, Sevanto S, Gallegos-Graves LV, Dunbar J. Biotic interactions are more important than propagule pressure in microbial community invasions. mBio. 2020;11(5):1-16. doi:10.1128/mBio.02089-20.

32. Stilianos L, Polz Martin F, Florent M, Albright MBN, Huber Julie HA, et al Function and functional redundancy in microbial systems Nature Ecology and Evolution 2018;2(6):936-943 doi:10.1038/s41559-018-0519-1.

33. Enke TN, Datta, MS, Schwartzman J, Cermak N, Schmitz D, Barrere J, Pascual-García A, Cordero OX. Modular Assembly of Polysaccharide-Degrading Marine Microbial Communities Current Biology 2019;29(9):1528-1535.e6 doi:10.1016/j.cub.2019.03.047.

34. Marsland, R., Cui, W. and Mehta, P. A minimal model for microbial biodiversity can reproduce experimentally observed ecological patterns.. Sci Rep. 2020;10, 3308. doi:doi.org/10.1038/s41598-020-60130-2. 
35. Pascual-García A, Bonhoeffer S, Bell T. Metabolically cohesive microbial consortia and ecosystem functioning. Philosophical Transactions of the Royal Society B: Biological Sciences. 2020;375(1798):0190245. doi:10.1098/rstb.2019.0245.

36. Altieri AH, Wesenbeeck BKV, Bertness MD, Silliman BR. Facilitation cascade drives positive relationship between native biodiversity and invasion success. Ecology. 2010;91(5):1269-1275. doi:10.1890/09-1301.1.

37. Li M, Wei Z, Wang J, Jousset A, Friman VP, Xu Y, et al. Facilitation promotes invasions in plant-associated microbial communities. Ecology Letters. 2019;22(1):149-158. doi:10.1111/ele.13177.

38. Machado D, Maistrenko OM, Andrejev S, Kim Y, Bork P, Patil KR, et al. Polarization of microbial communities between competitive and cooperative metabolism. Nature Ecology and Evolution. 2021;5(2):195--203. doi:10.1038/s41559-020-01353-4.

39. Livingston G, Jiang Y, Fox JW, Leibold MA. The dynamics of community assembly under sudden mixing in experimental microcosms. Ecology. 2013;94(12):2898-2906. doi:10.1890/12-1993.1.

40. Silva LP, Northen TR. Exometabolomics and MSI: Deconstructing how cells interact to transform their small molecule environment. Current Opinion in Biotechnology. 2015;34:209-216. doi:10.1016/j.copbio.2015.03.015.

41. Paczia N, Nilgen A, Lehmann T, Gätgens J, Wiechert W, Noack S. Extensive exometabolome analysis reveals extended overflow metabolism in various microorganisms. Microbial Cell Factories. 2012;11:1-14. doi:10.1186/1475-2859-11-122.

42. Yamagishi JF, Saito N, Kaneko K. Advantage of Leakage of Essential Metabolites for Cells. Physical Review Letters. 2020;124:048101. doi:10.1103/PhysRevLett.124.048101.

43. Yamagishi JF, Saito N, Kaneko K. Microbial Potlatch: Cell-level adaptation of leakiness of metabolites leads to resilient symbiosis among diverse cells. bioRxiv. 2020; p. 1-17. doi:10.1101/2020.11.06.370924.

44. Kurkjian HM, Javad Akbari M, Momeni B. The impact of interactions on invasion and colonization resistance in microbial communities. PLoS Computational Biology. 2021;17(1):1-18. doi:10.1371/journal.pcbi.1008643.

45. Acosta F, Zamor RM, Najar FZ, Roe BA, Hambright KD. Dynamics of an experimental microbial invasion. Proceedings of the National Academy of Sciences of the United States of America. 2015;112(37):11594-11599. doi:10.1073/pnas.1505204112.

46. Girvan MS, Campbell CD, Killham K, Prosser JI, Glover LA. Bacterial diversity promotes community stability and functional resilience after perturbation. Environmental Microbiology. 2005;7(3):301-313. doi:10.1111/j.1462-2920.2005.00695.x.

47. Eisenhauer N, Scheu S, Jousset A. Bacterial diversity stabilizes community productivity. PLoS ONE. 2012;7(3):1-5. doi:10.1371/journal.pone.0034517. 
48. Maron PA, Sarr A, Kaisermann A, Lévêque J, Olivier M, Guigue J, et al. High Microbial Diversity Promotes Soil Ecosystem Functioning. 2018;84:02738-17. doi:10.1128/AEM.02738-17.

49. Ratzke C, Barrere J, Gore J. Strength of species interactions determines biodiversity and stability in microbial communities. Nature Ecology and Evolution. 2020;4(3):376-383. doi:10.1038/s41559-020-1099-4.

50. Seebens H, Blackburn TM, Dyer EE, Genovesi P, Hulme PE, Jeschke JM, et al. No saturation in the accumulation of alien species worldwide. Nature Communications. 2017;8:1-9. doi:10.1038/ncomms14435.

51. Rillig MC, Tsang A, Roy J. Microbial community coalescence for microbiome engineering. Frontiers in Microbiology. 2016;7:1967. doi:10.3389/fmicb.2016.01967.

52. Calderón K, Spor A, Breuil MC, Bru D, Bizouard F, Violle C, et al. Effectiveness of ecological rescue for altered soil microbial communities and functions. ISME Journal. 2017;11:272-283. doi:10.1038/ismej.2016.86.

53. Wang JW, Kuo CH, Kuo FC, Wang YK, Hsu WH, Yu FJ, et al. Fecal microbiota transplantation: Review and update. Journal of the Formosan Medical Association. 2019;118(1):S23-S31. doi:10.1016/j.jfma.2018.08.011.

54. Wilson BC, Vatanen T, Cutfield WS, O'Sullivan JM. The super-donor phenomenon in fecal microbiota transplantation. Frontiers in Cellular and Infection Microbiology. 2019;9(2): doi:10.3389/fcimb.2019.00002.

55. Lindemann SR, Bernstein HC, Song HS, Fredrickson JK, Fields MW, Shou W, et al. Engineering microbial consortia for controllable outputs. ISME Journal. 2016;10(9): 2077-2084. doi:10.1038/ismej.2016.26.

56. Sung J, Kim S, Cabatbat JJT, Jang S, Jin Y, Jung GY, et al. Global metabolic interaction network of the human gut microbiota for context-specific community-scale analysis. Nat. Commun. 2017;8:15393. doi:10.1038/ncomms15393.

57. Tikhonov M, Monasson R. Collective phase in resource competition in a highly diverse ecosystem. Physical Review Letters. 2017;118:048103. doi:10.1103/PhysRevLett.118.048103.

58. Tikhonov M, Monasson R. Innovation rather than improvement: A solvable high-dimensional model highlights the limitations of scalar fitness. J. Stat. Phys. 2018;172:74-104. doi:10.1007/s10955-018-1956-6. 